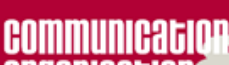
Organlofidin.

\section{Communication et organisation}

Revue scientifique francophone en Communication

organisationnelle

18 | 2000

Non-verbal, communication, organisation

\title{
La prise en compte de la multimodalité de la parole dans la description et analyse des conduites langagières
}

Jean-Marc Colletta

\section{OpenEdition}

Édition électronique

URL : https://journals.openedition.org/communicationorganisation/2427

DOI : 10.4000/communicationorganisation.2427

ISSN : $1775-3546$

Éditeur

Presses universitaires de Bordeaux

Édition imprimée

Date de publication : 1 novembre 2000

ISSN : 1168-5549

Référence électronique

Jean-Marc Colletta, « La prise en compte de la multimodalité de la parole dans la description et analyse des conduites langagières ", Communication et organisation [En ligne], 18| 2000, mis en ligne le 27 mars 2012, consulté le 05 août 2021. URL : http://journals.openedition.org/

communicationorganisation/2427 ; DOI : https://doi.org/10.4000/communicationorganisation.2427

Ce document a été généré automatiquement le 5 août 2021.

(c) Presses universitaires de Bordeaux 


\section{La prise en compte de la multimodalité de la parole dans la description et analyse des conduites langagières}

Jean-Marc Colletta

\section{Introduction}

1 L'objectif de la présente communication consiste à montrer qu'une approche multimodale ou «étho-linguistique » du dialogue et des conduites de discours oral est aujourd'hui possible.

2 Au préalable, qu'entend-on par approche «étho-linguistique»? Les conduites discursives sont des conduites complexes, que l'on peut tenter de saisir soit à un niveau global (comment réalise-t-on un récit ou un exposé théorique ? comment gère-t-on un entretien?), soit à un niveau local (comment performe t-on un acte de langage? comment exprime-t-on une position énonciative nuancée ? etc.). Les aspects formels et séquentiels de ces conduites ont été abondamment décrits au cours de ces trente dernières années par les linguistes s'intéressant à renonciation, à la structure des textes et à la conversation. Étant donné le caractère structurant de la parole et du matériau verbal dans ces conduites, on ne peut prétendre les décrire en faisant abstraction de leurs aspects linguistiques. Mais dès lors qu'on se situe dans une perspective multimodale, on ne peut non plus se satisfaire de descriptions et d'analyses se limitant aux seuls aspects verbaux. L'approche « linguistique » doit se doubler d'une approche " éthologique » où l'analyste s'attache à décrire aussi bien les aspects visuels et kinésiques de ces conduites que leurs aspects sonores, et se donne pour tâche de les analyser dans la pluri-sémioticité et la pluri-fonctionnalité qui les caractérise.

Ce point de vue est-il nouveau? Non, car cela fait un certain temps que les gestualistes s'intéressent à la communication parlée dans une optique multimodale, en interrogeant 
les relations entre la parole et le langage du corps. Toutefois, et pour des raisons que nous allons tenter d'analyser, force est de constater que nous connaissons encore bien peu de choses sur la manière dont la voix et le corps sont intégrés aux conduites discursives des locuteurs.

\section{Les linguistes et la question du non-verbal}

4 Force est de constater que jusqu'à une date très récente, et depuis la fondation de la linguistique comme science au début du $\mathrm{XX}^{\mathrm{e}}$ siècle, les linguistes se sont fort peu, voire pas du tout préoccupés des signifiants non verbaux. Le courant structuraliste, dominant durant toute la première moitié du siècle, a sans doute joué là un rôle négatif. En réduisant l'objet de la linguistique à la langue, définie comme un système formel abstrait, soit comme un objet purement théorique, les structuralistes, dans la lignée des réflexions de Saussure en Europe, de Bloomfield (et plus tard de son élève : Chomsky) aux États-Unis, ont écarté de leurs analyses tout ce qui avait rapport aux usages langagiers en contexte, soit à la parole. Dès l'instant où il n'y avait pas de place pour une linguistique de la parole, il ne pouvait y avoir de place pour une linguistique des signifiants non verbaux.

Cela dit, la linguistique a en parallèle connu une évolution considérable. Certains travaux marquants ont, dans la première moitié du siècle, posé les jalons de cette évolution.

6 Il en est ainsi des travaux d'anthropologues tels Mauss en France, Sapir et Malinowsky dans le monde anglo-saxon, qui ont attiré l'attention sur l'importance cruciale, tant au regard de la culture que de la formation de la personne, de l'échange social et de la communication interindividuelle ${ }^{1}$. Mais les travaux des sociologues de l'Ecole de Chicago ont également connu un retentissement important, ainsi que les réflexions de G.H. Mead, fondateur de «l'interactionnisme symbolique $»^{2}$.

7 Dans le champ des études linguistiques et littéraires, ce sont les travaux pionniers de Bakhtine-Volochinov en Union Soviétique qui vont avoir une influence prépondérante sur le développement des travaux interactionniste ${ }^{3}$ Les réflexions de Jakobson (1963) concernant la communication linguistique et les fonctions du langage, s'appuyant en même temps sur les apports des ingénieurs des sciences de l'information alors naissantes, auront également un grand retentissement en linguistique.

Enfin, en philosophie, certains apports feront date. Au tournant du siècle, ce sont par exemple la distinction opérée par Frege entre le « sens » et la « référence » des mots, et les réflexions de Peirce concernant l'indexicalité comme mode de signifiance. Ces réflexions vont avec d'autres contribuer à la naissance de la pragmatique, que Morris définit en 1938 comme une composante de la sémiotique. celle qui prend pour objet les relations entre les systèmes de signes (dont la langue) et leurs utilisateurs. Un peu plus tard, en 1945 Wittgenstein pose les bases de la philosophie du langage qui va donner naissance à la logique illocutoire et à la pragmatique contemporaine.

Les années 60 marquent un tournant, avec aux Etats-Unis l'apparition quasi simultanée de plusieurs courants :

- en sociologie, le courant ethnométhodologique d'une part, au sein duquel vont se développer les premiers travaux d'analyse conversationnelle ${ }^{4}$, et d'autre part 
l'approche originale de E. Goffman $(1973,1974)$, qui construit une microsociologie des échanges quotidiens extrêmement riche et influente ;

11 - dans le champ de l'ethnologie, l'ethnographie de la communication, qui entreprend de décrire les pratiques communicatives des individus dans divers contextes culturels ${ }^{5}$;

12 - en psychologie, ce sont les psychiatres de Palo Alto qui, s'appuyant sur les thèses de $G$. Bateson, développent une « axiomatique de la communication » au retentissement très important $^{6}$; dans le champ de la linguistique, W. Labov (1976) ouvre le champ à la problématique de la variation sociale des usages linguistiques. Tous ces travaux s'appuient sur des démarches très empiriques, et se caractérisent en conséquence par un très fort élan vers la description des pratiques communicatives et linguistiques effectives, dans les échanges quotidiens.

En France, c'est dans le champ même de la linguistique que s'opèrent des évolutions. Dans le sillage des travaux de linguistes tels Bally, E. Benveniste

$14(1966,1974)$ ouvre le champ de la linguistique à l'étude des marques de renonciation et théorise une vision nouvelle de la langue, qui doit être appréhendée dans ses usages effectifs, " en discours ». À la même époque, les philosophes anglo-saxons fondent la pragmatique des actes de langage ${ }^{7}$. La pragmatique linguistique va alors s'intéresser aux marques linguistiques de l'illocution (verbes performatifs, connecteurs), travaux dont on trouve abondamment trace aujourd'hui dans plusieurs revues, dont les Cahiers de Linguistique Française édités à Genève, la Revue de Sémantique et Pragmatique éditée à Orléans, ou encore, Pragmatics, la revue de l'International Pragmatics Association. En parallèle, se développent les travaux visant à décrire les textes écrits et les discours oraux, et les analyses concernant la structure, les aspects séquentiels de ces objets discursifs. Ces travaux de linguistique textuelle se sont abondamment nourris tout à la fois des études réalisées en psycholinguistique, et des apports des didacticiens de la langue écrite ${ }^{8}$.

On pourrait bien sûr évoquer d'autres travaux qui ont contribué à élargir le champ de la linguistique, tels ceux de Halliday (1978) en Grande-Bretagne, ou plus indirectement, de Bourdieu (1982) et Flahault (1978) en France. Il demeure que sous l'influence de ces apports très divers, la linguistique est passée progressivement de l'étude de la langue à l'étude des usages langagiers dans la communication et les discours. Le schéma page suivante représente cette ouverture vers des «sciences du langage » aux multiples objets. 


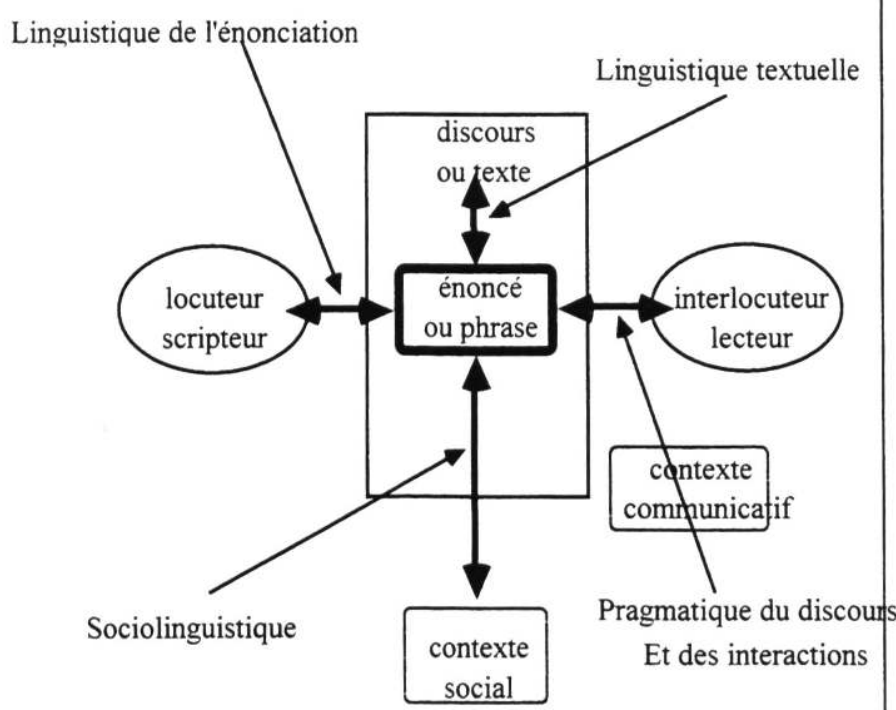

Les nouveaux courants en sciences du langage : élargissement de l'objet d'étude initial (la phrase) au texte, au contexte et aux partenaires de la communication.

Aujourd'hui, les spécialistes de la conversation et des discours oraux s'attachent à décrire les usages langagiers en contexte, les positionnements énonciatifs et les stratégies pragmatiques des locuteurs, et la structure des conversations et des discours. Ils ont à leur disposition les outils mis à jour en linguistique de renonciation, en pragmatique et en linguistique textuelle.

Toutefois ces marques, parfois analysées avec une grande finesse, sont toujours des marques de nature linguistique (opérateurs, connecteurs, marqueurs de constituants conversationnels, etc.). Aussi est-il temps de s'intéresser à d'autres marques, telles les marques prosodiques et kinésiques, si l'on veut avancer dans la description des discours et des interactions communicatives.

\section{Les gestualistes et la question de la multimodalité}

18 Les préoccupations relatives au langage du corps sont très anciennes. Mais le $\mathrm{XX}^{\mathrm{e}}$ siècle, avec la mise au point des techniques de cinématographie a vu se multiplier les travaux scientifiques portant sur la communication non-verbale et les gestes, mimiques, postures, regards et attitudes proxémiques (distances interindividuelles, conduites de toucher).

En 1969. S. Duncan distingue déjà 2 approches distinctes : une approche "structurale » ou descriptive, relevant des méthodes d'observation et d'analyse de l'éthologie et de la linguistique, et une approche "par les variables externes». relevant plutôt de la méthode utilisée en psychologie, où l'on cherche à établir des corrélations statistiques entre l'apparition ou la fréquence de conduites non-verbales et des variables psychologiques, sociologiques, culturelles, situationnelles ou autres.

Or lorsqu'on examine la littérature consacrée à la communication corporelle, force est de constater que la seconde approche a été nettement plus productive que la première. 
En témoignent aussi bien le contenu des principaux ouvrages de synthèse parus en France (J. Corraze 1980. P. Feyreisen et J.-D. De Lannoy 1985. M.A. Descamps 1989, J. Fast 1994) que la nature des publications qui paraissent dans le Journal of Nonverbal Behavior. Dans ce type d'approche, il y a à l'évidence peu de place pour une investigation des relations entre le langage du corps et les conduites verbales, puisque les variables mises en corrélation avec les mouvements corporels sont précisément des variables externes au flux communicationnel.

21 Toutefois, et dès les années soixante, apparaissent des travaux qui s'attachent à décrire les conduites non-verbales en elle-même et à explorer leurs liens aux conduites verbales. Concernant le premier point, on retiendra la tentative audacieuse de R.L. Birdwhistell $(1968,1972)$ d'appliquer la méthode structurale employée habituellement en linguistique à la description des gestes et mimiques. Concernant le second point, on rappellera le travail pionnier effectué par W.S. Condon et W.D. Ogston (1971) ${ }^{9}$ qui a permis de mettre à jour l'étroite synergie entre la parole et les mouvements corporels dans les conduites langagières, tant chez le locuteur (intra-synchronic) qu'entre les partenaires de la communication (inter-synchronie), ainsi que les fines analyses de A. Kendon $(1972,1980)$. qui permettent de mettre à jour des correspondances entre des unités (ou séquences) gestuelles et les unités (ou séquences) voco-verbales qu'elles accompagnent.

Par ailleurs, et en parallèle, s'est développé toute une réflexion sur les fonctions remplies par les gestes, regards, mimiques et postures dans la communication parlée. Dans un article de 1977. J. Cosnier s'est fait l'écho de cette réflexion en présentant les classifications élaborées par plusieurs auteurs (Efron. Mahl, Greimas, Friesen). D'autres ont été préposées, notamment par K. R. Scherer (1984), par J. Cosnier et ses collaborateurs (Cosnier 1977, 1993), par D. McNeill (1992) et plus récemment par nousmême (Colletta 1998). L'intérêt de ces classifications fonctionnelles de la gestualité, au même titre que les analyses sémiotiques très fouillées de G. Calbris (G. Calbris et L. Porcher 1989). tient à ce qu'elles focalisent l'attention sur les relations entre les signaux prosodiques et linguistiques de la parole et les signes non verbaux. Toutefois, elles ne sont pas forcément pertinentes lorsqu'on s'attaque à la description précise du flux pluri-sémiotique des conduites langagières, où d'autres paramètres que la fonction des mouvements corporels interviennent, notamment leur durée, leur mode d'accomplissement, ou leur position.

\section{L'approche étho-linguistique des conduites langières}

23 Ainsi, durant toute une période, les descriptions des spécialistes de la communication non-verbale n'ont guère croisé les analyses des linguistes, et la question des relations entre la parole et le langage du corps est restée au second plan. Pourtant, la parution de plus en plus fréquente d'ouvrages traitant explicitement de ces questions ou de travaux intégrant une perspective multimodale ${ }^{10}$ ", de même que le nombre croissant de colloques et rencontres consacrant tout ou partie de leurs travaux à la communication non-verbale ou à la multimodalité ${ }^{11} »$, montre que cet état de fait est en train d'évoluer, et que des rapprochements s'effectuent entre les gestualistes, les linguistes et phonéticiens, et également les psycholinguistes travaillant sur l'acquisition du langage.

Il est un fait qu'aujourd'hui : 
- on commence à bien connaître les fonctions remplies par les gestes, regards, mimiques et postures au regard des activités de parole, on étudie les processus iconiques et métaphoriques par lesquels les gestes servent d'outils pour construire dans l'espace les référents du discours, ou de signes kinésiques donnant une réalité spatiale à des concepts verbaux abstraits ${ }^{12}$,

- on commence à décrire les ressources corporelles en tant qu'elles permettent au locuteur de cadrer l'activité discursive en cours (de signaler qu'il commente ou ironise, qu'il fait une parenthèse ou une digression, qu'il reprend le fil de son récit, qu'il énonce un nouvel argument, etc. $)^{13} \ldots$

Ces données, associant les analyses des linguistes à la description fine des conduites non-verbales, offrent un regard nouveau sur la communication duelle. sur les conduites langagières orales en conversation et en discours, et pour ce qui concerne la perspective développementale, sur le développement de ces conduites chez l'enfant.

\section{BIBLIOGRAPHIE}

ADAM J-M., Les textes, types et prototypes. Nathan. Paris, 1992.

AUSTIN J.L., Quand dire c'est faire. Seuil, Paris, 1970.

BACHMAN C, LINDENFELD J., SIMONIN J., Langage et communications sociales, Credif-Hatier, Paris, 1981.

BAKHTINE M., VOLOCHINOV N., Le marxisme et la philosophie du langage, Minuit, Paris, 1977.

BENVENISTE E., Problèmes de linguistique générale, T. 1 et T.2., Gallimard, Paris, 1966, 1974.

BERNICOT J. et al.. De l'usage des gestes et des mots chez l'enfant, Armand Colin, Paris, 1998.

BIRDWHISTELL R.L., « L'analyse kinésique », Langage n 10. 1968, pp. 101-106.

BIRDWHISTELL R.L., « A kinesic-linguisitc execrise: the cigarette scène », in Gumperz et Hymes, Directions in sociolinguistics, 1972, pp. 381-404. Bourdieu P., Ce que parler veut dire. Fayard, Paris, 1982. Bronckart J-P., Activité langagière, textes et discours. Pour un interactionnisme socio-discursif, Delachaux et Niestlé, Neuchâtel, 1996. Calbris G., Porcher J-L., Geste et communication, CredifHatier, Paris, 1989. Colletta J-M., « Les conduites narratives chez l'enfant : approche étholinguistique et développementale », communication au Vie Colloque de l'IPrA, Reims, juillet 1998, à paraître dans le $n^{\circ} 22$ de la revue Lidil, Grenoble.

COLLETTA J-M., " Catégorisation fonctionnelle des kinèmes. Etude autour d'un outil d'analyse », communication au Colloque Ora'ge. Besançon, déc. 1998. Colletta J-M., «Quand les enfants argumentent avec les mots, la voix et le corps », communication au Colloque International Les relations intersémiotiques, Lyon, déc. 1999. Actes à paraître.

CONDON W.S., « Une analyse de l'organisation comportementale », in Cosnier et Brossard, La communication non-verbale, 1984, pp. 31-70. Condon W.S., Ogston W.D., « Speech and body motion synchrony of the speaker hearer ", in Horton \& Jenkins, The perception of language, Charles Merrill, Clombus. 
CORRAZE J., Les communications non-verbales, P.U.F., Paris, 1980.

COSNIER J., C Communication non-verbale et langage », Psychologie

médicale. $\mathrm{n}^{\circ}$ 9-11. 1977, pp. 2033-49.

COSNIER J., « Etude de la mimogestualité », in R. Plety, Ethologie des communications humaines : aide-mémoire méthodologique, 1993, pp. 103-115.

COSNIER J., BROSSARD A., La communication non-verbale, Delachaux \& Niestlé, Neuchâtel, 1984.

DESCAMPS M-A., Le langage du corps et la communication corporelle, P.U.F.. Paris, 1989.

DUNCAN S., « Non-verbal communication ». Psychological Bulletin $\mathrm{n}^{\circ}$ 72-2, 1969, pp. 118-137.

FAST J., Le langage du corps, Eds. de L'homme, Paris, 1994.

FEYEREISEN P., De Lannoy J-D.. Psychologie du geste, Pierre Mardaga, Bruxelles, 1985.

FLAHAUT F., La parole intermédiaire, Seuil. Paris. 1978.

GOFFMAN E., La mise en scène de la vie quotidienne (2 tomes), Minuit, Paris,

1973,1974 .

GOODWIN C, Conversational organization. Academic Press, London, 1981.

GUMPERZ J.J., HYMES D., Directions in sociolinguistics: the ethnography of communication, Holt, Rinehart and Winston, Chicago, 1972.

GRICE H.P., « Logique et conversation », Communication n³0, 1979, pp. 57-72.

HALLIDAY M.A.K.. Language as social semiotic, Edward Arnold, London, 1978.

KENDON A., "Some relationships between body motion and speech », in Siegman \& Pope, Studies in dyadic communication, Pergamon, New-York, 1972.

KENDON A., " Gesticulation and speech, two aspects of the process of utterance ", in M.R. Key. The relationship of verbal and nonverbal communication, 1980. pp. 207-227.

KENDON A.. Conducting interaction. Patterns of behavior in focused encounters, Cambridge University Press, Cambridge, 1990.

KEY M. R. (éd.), The relationship of verbal and nonverbal communication. Mouton, The Hague, 1980.

LABOV W., Sociolinguistique, Minuit. Paris, 1976.

LÉONARD J-L., PINHEIRO M-B.. « Enonciation et non-verbal : aspects de la cohésion linguistique dans un récit oral poitevin ». Langage et société n 65, 1993, pp. 39-68.

LEVINSON S., Pragmatics, Cambridge University Press, Cambridge, 1983. Mauss M., Sociologie et anthropologie, P.U.F.. Paris, 1985.

Mcneill D.. Hand and mind. What gestures reveal about thought, The University of Chicago Press, Chicago. 1992.

mead G. H., Mind, self and society from the standpoint of a social behaviorist, The University of Chicago Press. Chicago. 1967. MORRIS C.W., « Fondements de la théorie des signes », Langage n 35. 1974, pp. 15-21.

PLETY R. (éd.), Ethologie des communications humaines : aide-mémoire méthodologique, ARCI et P.U.L., Lyon, 1993. 
POYATOS F. (éd.), Advances in nonverbal communication, John Benjamins Publishing Company, Amsterdam, 1992.

SANTI S., GUAÏTELLA L, CAVE C, KONOPCZYNSKI G. (eds.). Oralité et gestualité. Communication multimodale, interaction. L'Harmattan, Paris, 1998. Sapir L., Anthropologie. 1. Culture et personnalité. Minuit. Paris, 1967. Saville-Troike M., The ethnography of communication. Basil Blackwell, Oxford, 1982.

SCHERER K. R., "Les fonctions des signes nonverbaux dans la conversation », in Cosnier et Brossard, La communication non-verbale, 1984, pp. 71-100. Searle John R., Les actes de parole, Hermann, Paris, 1972. Streek J., Knapp M-L., « The interaction of verbal and visual features in human communication ", in F. Poyatos, Advances in nonverbal communication, 1992, pp. 3-23.

WATZLAWICK P., HELMICK B. J., JACKSON D. D.. Une logique de la communication. Seuil coll. Points, Paris, 1972.

WIDMER J., Langage et action sociale, Editions Universitaires, Fribourg, 1986.

WIENER M., Shilkret R., Devoe S., « Acquisition of communicative competence: is language enough?», in M.R. Key, The relationship of verbal and nonverbal communication, 1980, pp. 275-294.

WINKIN Y., La nouvelle communication. Seuil, Paris. 1981.

WITTGENSTEIN L., Les investigations philosophiques, Gallimard, Paris, 1961.

\section{NOTES}

1. Voir M. Mauss 1985 (éd. orig. 1902-38), E. Sapir 1967 (ed. orig. 1917-38).

2. G.H. Mead 1967 (éd. originale 1934).

3. M. Bakhtine-N. Volochinov 1977 (ed. orig. 1929).

4. Voir à ce sujet C. Bachman, J. Lindenfeld, J. Simonin 1981. J. Widmer 1986, ou encore S. Levinson 1983.

5. Voir l'important ouvrage édité par J.J. Gumperz et D. Hyines en 1972, et pour une synthèse ultérieure : M. Saville-Troike 1982.

6. Voir le célèbre ouvrage de P. Watzlawick, J.H. Beavin et Don D. Jackson paru en 1972, ainsi que le recueil de textes édité par Y. Winkin en 1981.

7. Parmi les travaux fondateurs : J.L. Austin 1970, J.R. Searle 1972, HP. Grice 1979.

8. Voir notamment J.-M. Adam, J.-M. Adam, Les textes : types et prototypes, Paris, Nathan. 1992 et J.P. Bronckart, Activité langagière, textes et discours, pour un interactionnisme socio-discursif, Delachaux et Niestlé. (1996).

9. Voir également W.S. Condon (1984).

10. M.R. Key 1980, A. Kendon 1990, F. Poyatos 1992, D. Mc Neill 1992, J. Bernicot et al. 1998.

11. Quelques faits significatifs : les deux derniers colloques de l'International Pragmatic Association ont ouvert des ateliers consacrés aux aspects non verbaux de la communication, ce qui montre l'intérêt croissant des linguistes pour les questions relatives au non-verbal ; un colloque organisé à Besançon en 1998 (le colloque Ora'ge) était explicitement consacré à la multimodalité ; enfin, le bulletin Kinélogos édité par J. Cosnier, G. Calbris et R. Pléty annonce régulièrement de nouvelles rencontres autour de ces thèmes.

12. Voir notamment les analyses de G. Calbris (1989) et de D. McNeill (1992).

13. Concernant ce point, on se reportera aux analyses de C. Goodwin (1981) et de A. Kendon (1990), ainsi qu'aux fines analyses multimodales des conduites langagières d'adultes en 
conversation, analyses conduites par J.-L. Léonard et M.-B. Pinheiro (1993), ou encore, J. Streeck et M.-L. Knapp (1992). Pour ce qui concerne la description multimodale des conduites narratives et argumentatives chez l'enfant, on se reportera à J.-M. Colletta $(1998,1999)$. Concernant le récit, une analyse développementale de ces conduites entre 6 ans et 11 ans (J.-M. Colletta 1998) a fait apparaître que leur évolution affectait simultanément l'ensemble des dimensions de la parole : plus les conduites verbo-narratives se complexifient, plus les usages voco-intonatifs, gestuels, mimiques et posturaux de l'enfant s'affinent et contribuent au cadrage et à l'illustration du récit mis en mots. Les aspects linguistiques, prosodiques et kinésiques sont donc intimement liés dans la réalisation des conduites narratives, et en conséquence, sont affectés en même temps par les évolutions en cours chez l'enfant.

\section{AUTEUR}

JEAN-MARC COLLETTA

Lidilem, IUFM et Université Stendhal, Grenoble 